\title{
MicroED: Conception, Practice and Future Opportunities
}

\author{
Emma Danelius and Tamir Gonen
}

HHMi/UCLA, Los Angeles, California, United States

My laboratory studies the structures of membrane proteins that are important in maintaining homeostasis in the brain. Understanding structure (and hence function) requires scientists to build an atomic resolution map of every atom in the protein of interest, that is, an atomic structural model of the protein of interest captured in various functional states. In 2013 we unveiled the method Microcrystal Electron Diffraction (MicroED) and demonstrated that it is feasible to determine high-resolution protein structures by electron crystallography of three-dimensional crystals in an electron cryo-microscope (CryoEM). The CryoEM is used in diffraction mode for structural analysis of proteins of interest using vanishingly small crystals. The crystals are often a billion times smaller in volume than what is normally used for other structural biology methods like x-ray crystallography. The electron beam is focused on a target crystal in diffraction mode and data collected using a fast camera in movie mode as the crystal is continuously rotated in a single direction. Data are then processed using standard X-ray crystallography software including, indexing, background substraction, scaling and integration. Phasing can be achieved by molecular replacement, direct methods, using heavy metals and radiation damage or by recording high resolution images and extracting phase information from there. Phase extension procedures as published previously can be used if necessary.

In this seminar I will describe the basics of this method, from concept to data collection, analysis and structure determination, and illustrate how samples that were previously unattainable can now be studied by MicroED. I will conclude by highlighting how this new method is helping us understand major brain diseases like Parkinson's disease; helping us discover and design new drugs; shedding new light on chemical synthesis and small molecule chemistry; and showing us unprecedented level of details with sub atomic resolutions.

\section{References}

Nannenga, Brent L; Gonen, Tamir; The cryo-EM method microcrystal electron diffraction (MicroED) Journal Article; Nat. Methods, 16 (5), pp. 369-379, 2019.

Shi, Dan; Nannenga, Brent L; Iadanza, Matthew G; Gonen, Tamir; Three-dimensional electron crystallography of protein microcrystals Journal Article; Elife, 2 , pp. e01345, 2013.

Nannenga, Brent L; Shi, Dan; Leslie, Andrew G W; Gonen, Tamir; High-resolution structure determination by continuous-rotation data collection in MicroED Journal Article; Nat. Methods, 11 (9), pp. 927-930, 2014.

Wisedchaisri, Goragot; Gonen, Tamir; Fragment-Based Phase Extension for Three-Dimensional Structure Determination of Membrane Proteins by Electron Crystallography Journal Article, Structure, 19 (7), pp. 976-987, 2011. 